\title{
La perception au fondement de la connaissance. Les enseignements d'une ingénierie représentationnelle ternaire
}

\author{
Jean-Pierre Bréchet ${ }^{1}$, Gérard Gigand ${ }^{2}$ \\ 1 Sciences de gestion, Université de Nantes, IEMN-IAE, LEMNA, 44322 Nantes, France \\ 2 Sciences de l'éducation, Complexitude, 91150 Ormoy-la-Rivière, France
}

\begin{abstract}
Toute connaissance procède de la perception que nous avons du monde. D'où l'importance extrême de comprendre les caractéristiques de la cognition de la réalité phénoménologique dans laquelle elle nous engage. Cette nécessité s'impose tout particulièrement à la recherche, dont l'objectif est précisément d'introduire la plus grande rigueur possible dans l'acte de connaître. Cette préoccupation est à l'origine de nombreuses réflexions philosophiques. Mais elle est aussi l'objet de réflexion des plus grands chercheurs eux-mêmes, qui s'efforcent ainsi de comprendre, à partir de leur pratique, le processus de production de connaissances qu'ils mettent en œuvre. Ils visent, ce faisant, tout à la fois à être mieux en mesure d'évaluer euxmêmes les limites des connaissances qu'ils apportent, et à mieux armer le chercheur en lui révélant l'outillage intellectuel qu'il utilise implicitement dans sa quête de compréhension de l'existant. C'est en ce sens que l'on peut parler d'une " ingénierie » de la recherche. Il n'est pas surprenant que cette démarche conduise les auteurs à s'ancrer dans la question de la complexité et, partant, à examiner les voies pratiques tentées pour y répondre, avec la transdisciplinarité comme horizon. Ils fournissent ainsi tout un appareillage intellectuel qui ouvre l'esprit au décloisonnement entre disciplines, et qui fournit les concepts et l'ossature d'ensemble nécessaires pour le penser et le conduire de façon rigoureuse.
\end{abstract}

La Rédaction

\section{Mots-clés :}

réel ; réalité ;

complexité ;

épistémologie ;

perception ; transdisciplinarité

\begin{abstract}
Résumé - Ce texte aborde la question de l'accès cognitif à la réalité phénoménologique à partir de la reconnaissance du jeu de trois invariants (l'incomplétude, l'autoréférence et l'indétermination) qui conditionnent notre rapport conscient au monde. La lecture ternaire ainsi proposée du processus d'observation consciente qu'engage la perception conduit à poser que toute perception est à la fois partielle, partiale et parcellaire. L'intelligibilité de la réalité s'inscrit alors dans un référentiel qui articule domaine de validité, champ d'intelligibilité et degré de résolution. En même temps, elle confronte la réflexion à la Relativité générale, à l'Humain et à l'Organisation comme constituants de la réalité phénoménologique et du regard pluri, inter et spécifiquement transdisciplinaire.
\end{abstract}

\begin{abstract}
Perception as the founding component of knowledge. The teachings of a ternary approach through a graphic representation. This article deals with the issue of accessing phenomenological reality. "Accessing" is a founding concept of the paradigm of complexity and constructivism. Here, the perception phenomenon throws a new light on the issue of accessing. The first step is to recognize the presence of three constants at the heart of our conscious relationship to our environment at large: incompleteness, self-reference and indeterminacy. The proposition here is to approach the concept of perception under the ternary guideline of transdisciplinarity. This leads us to the observation that perception is definable within three forms of the concept of Limit: These are partial (in deficit), partial (biased) and partial (fragmented). The challenge is then to be able to launch a combination process of antagonistic relationships between the three binary pairs of poles consisting of partial (in deficit) with partial (biased), partial
\end{abstract}

\footnotetext{
Auteur correspondant : J.-P. Bréchet, jean-pierre.brechet@univ-nantes.fr

Pour en savoir plus sur Complexitude, vous pouvez consulter le site http://www.complexitude.org/ ou contacter G. Gigand (gerard.gigand@complexitude.org).
} 
(biased) with partial (fragmented) and partial (fragmented) with partial (in deficit). This analysis bearing on ternary relations is called "trialectic". It is by no means esoteric and we believe that it opens up a fruitful understanding for a pluri or trans disciplinary thinking. This approach allows for contradictions and antagonisms which are intrinsically part of a dynamic comprehension of reality. A trialectic analysis of perception reveals a close relationship to the development of knowledge and the phenomenology of reality as such. The intelligibility of reality emerges from a system of references combining dynamically three entities namely a sphere of validity, a field of intelligibility and a degree of resolution. Simultaneously, the trialectic approach will involve general relativity, the human entity and the principle of organization as basic constituents of the phenomenon of reality and of the transdisciplinary approach.

\section{Introduction}

La complexité n'est pas une propriété des objets, mais une qualification que nous attribuons à la réalité que nous percevons et que nous interprétons. Cette idée affirmée de longue date dans le courant de la pensée complexe ou du constructivisme ${ }^{1}$ engage immédiatement à s'intéresser à la réalité phénoménologique, à laquelle la perception nous donne accès et sur laquelle nous pouvons alors exercer notre raison. Il est dès lors souhaitable d'abandonner toute référence au Réel (voir Encadré 1) posé comme inconnaissable en lui-même. $C^{\prime}$ est à la discussion de ce point de vue qu'est consacré le texte qui suit ${ }^{2}$.

$\mathrm{Au}$ fondement de l'approche proposée ici, on trouve donc la question de la perception et de l'intelligibilité qui se construit sur cette perception première. Mioara Mugur-Schächter (2012), physicienne quantique, synthétise bien la posture dans laquelle nous nous inscrivons et qu'elle qualifie de constructive : « Les représentations scientifiques ne sont nullement des découvertes d'un réel qui serait et qu'il faudrait découvrir, mais elles sont toujours des constructions fondées sur des interactions entre, d'une part, les perceptions et les modes de penser et d'opérer des observateurs-concepteurs, et, d'autre part, un réel physique dont la manière d'être « en soi » est foncièrement et définitivement hors du connaissable parce qu'elle est inextricablement fondue dans le produit de ces interactions ${ }^{3} »$.

Notre contribution peut être considérée comme l'expression d'une posture constructiviste en épistémologie et d'un ancrage dans la phénoménologie en

\footnotetext{
1 On mentionnera simplement ici, à titre de repère dans la francophonie, les travaux fondateurs de Jean Piaget (1967, 1970), d'Edgar Morin (La méthode, les trois premiers volumes, parus en 1977, 1980 et 1986) et de Jean-Louis Le Moigne (1990, 2006).

2 Cette contribution est le fruit d'un travail de recherche mené pendant plus de trois ans par les auteurs à partir de la proposition d'une méthode ternaire, dite trialectique, par Gérard Gigand $(2007,2010)$. L'intégralité du travail de recherche mené est à paraître dans un livre préfacé par E. Morin.

3 In Genelot et Avenier (2012, p. 14-15). Cf. aussi l'ouvrage majeur de M. Mugur-Schächter sur le métissage des connaissances (2006).
}

philosophie. Mais dans le cadre de ce repérage large, il faut immédiatement préciser que notre propos ne se veut pas de nature philosophique, mais de nature méthodologique. Il est centré sur l'élucidation du processus de perception. Ce processus, par ses propriétés, rend explicite le fait que nous construisons la connaissance sur « l'inconnaissabilité » radicale qu'engage notre présence au monde. Nous proposons d'appeler « ingénierie représentationnelle » l'outillage que nous utilisons pour procéder à cette construction, qui naît nécessairement de notre rapport au monde physique et idéel et nous aide à l'établir. Cette « ingénierie » prend une importance toute particulière dans le travail de recherche. Nous allons donc plus particulièrement nous attacher à construire une grille d'analyse des processus de connaissance qui sont à l'arrière-plan de tout travail de recherche. À cet égard, on peut considérer que cet article nourrit la réflexion épistémologique sur le dépassement des cloisonnements disciplinaires, en ce sens qu'y est engagé le rapport premier aux objets d'étude (phénomènes ou concepts) et à leur compréhension. Il devrait notamment permettre de comprendre ce que recouvre la notion de point de vue, dont on sait l'importance qu'elle a dans la pratique et la compréhension de l'interdisciplinarité. Si l'on admet, avec E. Morin, que la pensée occupe une position seconde (méta-), et qu'elle organise la connaissance et les logiques qui la fondent (Morin, 1986), la posture réflexive qui est proposée dans ce texte constitue autant une contribution à une épistémologie de la pensée qu'à une épistémologie de la connaissance.

Nous partirons du paradigme de la complexité pour identifier les trois invariants qui sont pour nous constitutifs de l'existence au monde: celui de l'« incomplétude », celui de $l^{\prime}$ « autoréférence » et celui de l'« indétermination» (1). Nous envisagerons ensuite le processus d'observation consciente qui conduit à comprendre la perception comme toujours partielle, partiale et parcellaire (2). De ce fait, la connaissance s'inscrit dans un référentiel qui recouvre un domaine de validité des connaissances, un champ d'intelligibilité des phénomènes et un degré de résolution de l'observation, en même temps qu'elle confronte à la transdisciplinarité, comprise comme mêlant des considérations ayant trait aux sphères de la Relativité générale, de l'Humain et de l'Organisation (3). 


\section{Encadré 1. La « réalité » n'est pas le « Réel »}

Un point essentiel pour la compréhension de cet essai concerne l'approche du « Réel » et de la « réalité ». Après des siècles, ces notions restent fondamentalement problématiques. Le Réel est ici entendu comme échappant intrinsèquement à notre capacité d'observation. Le Réel est considéré comme le Tout (le Un) qui ne peut être observé comme tel, précisément par le fait, pour les observateurs que nous sommes, que notre présence distincte qui est en même temps une appartenance ou une inhérence, empêche le Réel d'être un Tout et d'être percevable comme tel, puisque nous en faisons partie. Prétendre observer et même prendre en compte le «Réel» dans un raisonnement, c'est prétendre à l'accessibilité de la cause première. Pour le dire simplement, personne ne constatera le début ou la fin du monde. Nous avons donc choisi, comme d'autres auteurs, de ne rien dire du " Réel », de ne pas le qualifier, faute de pouvoir en connaître la moindre propriété (qu'il aurait en propre, en dehors d'une interaction avec nous). Il ne saurait avoir des propriétés sans devenir une "réalité », c'est-à-dire sans qu'il soit perçu et interprété par nous avec nos instruments. Nous saisissons le monde, dont on ne nie pas l'existence, par l'interprétation que nous en formons, c'està-dire la réalité phénoménologique. Pour ces raisons, nous écrivons le « Réel » avec un « R » majuscule et la « réalité » avec un « $\mathrm{r}$ » minuscule. De plus, étant donné leur valeur générique, nous supprimons les guillemets dans le cours du texte.

\section{L'inconnaissabilité au fondement de la connaissance}

La complexité, que l'on associe ici aux importants travaux de synthèse et d'approfondissement d'Edgar Morin ( $c f$. les six tomes de La méthode), vaut pour notre entendement, non pour le monde observé ou les objets, dont elle serait un attribut. Elle est une propriété du regard que l'on porte sur eux. L'ancrage de notre propos dans le paradigme de la complexité est indispensable pour traiter de la correspondance entre la pensée et l'univers phénoménal (celui auquel renvoie le concept de réalité phénoménologique). C'est le point de départ permettant d'identifier les trois grands invariants déjà évoqués (l'incomplétude, l'autoréférence et l'indétermination) qui sont constitutifs du rapport au monde à la fois, en ce qu'ils expriment l'existence en même temps que sa finitude, l'impossibilité radicale de connaître en même temps que sa possibilité.

\section{L'ancrage dans le paradigme de la complexité}

La pensée de la complexité ne peut s'imaginer sans le rapprochement, mais aussi sans le dépassement, des savoirs disciplinaires. Pourtant, cet enrichissement souhaité n'est nullement la norme dans l'univers de production des connaissances car, comme nous le rappelle souvent $\mathrm{E}$. Morin, la science s'est sectorisée entre les trois grands champs de la connaissance que sont la physique, la biologie et les sciences humaines et, à l'intérieur de ces champs, le compartimentage a poursuivi son œuvre sous le poids des logiques de spécialisation des savoirs ${ }^{4}$. La complexité appelle la transdisciplinarité, qui ne doit pas simplement être un multiple ou un entre de la disciplinarité comme a su l'affirmer Basarab Nicolescu $\left(2002^{5}\right)$. On peut aisément adhérer à ces évocations rapides, mais il demeure néanmoins difficile de dépasser le simple rapprochement des regards disciplinaires, et de disposer d'une méthode pour ce faire. À bien des égards, notre proposition méthodologique tente de faire vivre une perspective transdisciplinaire.

La pensée de la complexité est une pensée de l'acceptation et de l'intégration des contradictions. Abordant la question de la contradiction, E. Morin (2009) nous en rappelle le surgissement au cœur des interprétations de la réalité, lorsqu'un objet physique peut être saisi sous sa réalité ondulatoire ou corpusculaire. Pour cet auteur, cette contradiction de la logique, que l'on retrouve aussi dans la logique formelle (le paradoxe du Crétois ${ }^{6}$, la faille de consistance de Gödel ${ }^{7}$ ), ne peut être levée que par l'introduction d'un méta point de vue complexe. Différentes perspectives « ternaires » trouvent ici leur point d'ancrage. Ainsi, selon E. Morin, la pensée dialogique permet de saisir à la fois les complémentarités et les antagonismes en introduisant la possibilité du tiers inclus ${ }^{8}$. Jean-Jacques Wunenburger (1990) parle d'une « raison contradictoire ", qui ne s'épuise nullement dans la raison dialectique, tout au contraire, puisque toute idée de réconciliation est repoussée. Pour ces deux auteurs, comme pour bien $\mathrm{d}^{\prime}$ autres ${ }^{9}$, la saisie des phénomènes doit maintenir une compréhension dynamique (" énergétique »). Dans le dépassement possible des lectures dichotomiques ou binaires par une approche ternaire,

4 Cf. Bréchet (2012) pour une synthèse.

5 Cf. aussi le site internet qu'il anime sur la transdisciplinarité, http:/ / ciret-transdisciplinarity.org/index.php.

6 On cite souvent Euboulide de Milet : « Tous les Crétois sont des menteurs, c'est Épiménide le Crétois qui le dit ».

7 Kurt Gödel, en 1931, énonce que tout formalisme logique qui comprend l'arithmétique repose sur des assertions indécidables ou indémontrables, et pour certaines contradictoires.

8 Nous introduirons la notion de tiers incluant, la préférant à celle de tiers inclus dans notre réflexion.

9 On pense ici à des auteurs comme Stéphane Lupasco, Basarab Nicolescu, Yves Barel, Élie Bernard-Weil (dont les positions ne sont pas pour autant les mêmes) ou bien encore, dans un autre registre, à François Jullien (à propos de la pensée chinoise) ; mais nous ne pouvons que les évoquer dans le cadre de cet article. 
nous retrouverons de tels ingrédients, mais la question méthodologique reste toujours largement ouverte. Notre proposition associe une lecture ternaire et le jeu de trois binômes identifiés comme constitutifs du complexe ternaire.

Il faut enfin prendre la mesure de la correspondance entre la pensée et l'univers phénoménal que recouvre le concept de réalité phénoménologique. E. Morin (1986, 2009) nous rappelle que la pensée retrouve dans la réalité qu'elle perçoit sa propre complexité qui est elle-même le produit d'une faculté humaine issue d'un processus de complexification au sein du monde. L'accès à la réalité, donc à la connaissance, advient en même temps que l'existence au monde. L'accès en tant que tel est le maître mot pour désigner ce qui se joue pour des êtres confrontés à la finitude sur le plan tant de leur vie que de leur connaissance, mais riches en même temps de cette finitude qui est manifestation de la vie et permet $l^{\prime}$ accès ${ }^{10}$.

L'accès est pour nous la clé de voûte de l'ingénierie ternaire que nous proposons et que nous disons « trialectique » pour la qualifier au mieux de notre point de vue. Elle prend appui sur la reconnaissance des trois grands invariants de l'incomplétude, de l'autoréférence et de l'indétermination, qui manifestent, chacun, à la fois l'Être et le Connaître dans une inséparabilité fondamentale.

\section{En quête des invariants du rapport au monde : l'incomplétude, l'autoréférence, l'indétermination}

De nombreux auteurs, philosophes ou penseurs de l'homme dans ses facettes existentielles ou religieuses ont souligné le mystère de la vie et de la naissance au monde: incomplétude radicale qui a conduit Leibniz, comme Descartes et bien d'autres, à construire une représentation de Dieu comme nécessité logique. L'incomplétude, c'est la reconnaissance de la finitude radicale de l'homme en même temps que celle d'une finitude tout aussi radicale de la connaissance qui accompagne la faculté de connaître. Dès le $\mathrm{XV}^{\mathrm{e}}$ siècle (en 1440 exactement), Nicolas de Cues (2013) avait su remarquablement exprimer ces idées, en se nourrissant d'ailleurs des réflexions antérieures de plus de cent cinquante ans de Maître Eckhart : il voyait dans la « Docte Ignorance » l'attitude digne de l'homme soucieux de sa propre possibilité de connaître face à l'infini. L'incomplétude se présente comme un invariant, comme une constante de notre existence, qui nous interdit d'imaginer l'accès à une vérité

\footnotetext{
10 Par « accès ", on entend habituellement le fait de parvenir à un lieu ou de disposer d'un objet. Ici, le terme renvoie à une idée de médiation, à un processus en soi n'ayant aucune visée $\mathrm{d}^{\prime}$ «appropriation ». L'intentionnalité est sur le processus et non sur l'acquisition. Toute question est un accès, l'exercice trialectique est un accès.
}

absolue, totale, en même temps qu'elle manifeste que l'homme existe : "Si nous avions accès à notre inconnu, $c^{\prime}$ est donc que nous serions omniscient, omniprésent au monde qui serait nous, l'idée même de notre existence au monde n'aurait plus de sens » (Morin, 2009, p. 7).

$\mathrm{Du}$ fait de notre existence, qui est une présence physique (biologique, psychologique...) au monde, se manifestent plusieurs formes de compréhension de l'incomplétude. Selon nous, celle-ci peut se comprendre comme l'expression de trois phénomènes liés, mais que l'on peut distinguer. Tout d'abord, la présence spatio-temporelle elle-même (qui pourrait se traduire par l'idée que l'observateur est dans le tableau) entraîne une cécité ou point aveugle radical qui nous accompagne et qui empêche la connaissance d'être totalisante. En lien encore avec notre présence physique dans l'espace et le temps, l'existence d'un angle mort manifeste l'importance de la localisation de l'observateur. Enfin, les processus mêmes qui manifestent la vie sont une autre source d'incomplétude, dynamique celle-là, résultant de l'inertie croissante qu'ils comportent en eux-mêmes et qui les empêchent d'atteindre un accomplissement total. Toute dynamique engendre son impossibilité de perdurer indéfiniment et engage ainsi un troisième phénomène, propre à l'incomplétude, que nous dénommons « la taille critique ». En résumé, l'incomplétude est un invariant de notre rapport au monde qu'au stade actuel de notre réflexion, nous voyons s'exprimer dans trois formes radicales de limites à notre capacité de connaître : le point aveugle (une incomplétude structurelle), l'angle mort (une incomplétude situationnelle), la taille critique (une incomplétude processuelle).

Comme le rappelle souvent E. Morin, l'intelligence de la complexité ne sera jamais la quête d'une quelconque complétude synonyme d'exhaustivité. La multiplication des regards et des analyses ne saurait tenir lieu de solution à cette incomplétude fondamentale et indépassable. Les trois déclinaisons que nous venons d'identifier sont elles-mêmes reliées, c'est-à-dire qu'elles sont à la fois singulières et causalement inséparables.

Nous pouvons également nous appuyer sur nombre d'auteurs tels que Kant, Lévinas, Pascal ou Descartes, pour introduire la référence à l'autoréférence et l'importance que nous lui accordons. Descartes, par exemple, l'envisage - non sans lien avec l'incomplétude - liée au fait même d'exister : "La faculté d'imaginer qui est en moi, et de laquelle je vois par expérience que je me sers lorsque je m'applique à la considération des choses matérielles, est capable de me persuader de leur existence : car quand je considère attentivement ce que c'est que l'imagination, je trouve qu'elle n'est autre chose qu'une certaine application de la faculté qui connaît, au corps qui lui est intimement présent, et partant qui existe » (Descartes, 2009, p. 180). Quand il nous dit «je pense donc je suis », ne nous livre-t-il pas d'ailleurs l'expression 
la plus affirmée possible de l'autoréférence? Celle-ci manifeste le pouvoir de voir ce que l'on veut voir, une intentionnalité chevillée au corps. C'est ainsi qu'elle est mise au fondement même de l'existence humaine par le courant existentialiste depuis Franz Brentano, Edmund Husserl, Alfred Schütz ou, plus près de nous, Jean-Paul Sartre ou Maurice Merleau-Ponty.

L'autoréférence s'affirme donc bien comme un deuxième invariant engagé dans tout avènement au monde physique et à la connaissance. Exprimant le point de vue, la subjectivité, l'éprouvé, cet invariant prend logiquement trois formes que suggère assez facilement la littérature et que notre travail nous a conduits à identifier clairement : les phénomènes d'autopersuasion, d'implication et d'appropriation. L'autopersuasion est $\mathrm{d}^{\prime}$ ordre structurel, en ce sens qu'une structure observatrice ne peut survivre, c'est-à-dire s'adapter, qu'en postulant comme vraie l'interprétation qu'elle fait de son environnement. C'est l'autoréférence structurelle. L'implication, telle que nous la considérons ici, n'est pas le choix de s'impliquer ou de ne pas s'impliquer ; elle procède purement et simplement du constat d'une présence, d'une existence, elle exprime l'idée d'une inéluctable implication de fait, d'un état d'implication dans la situation vécue. C'est l'autoréférence situationnelle. L'appropriation, quant à elle, engage une temporalité, suppose un inventaire des éléments appropriables qui se réalise nécessairement dans un processus contextualisé. C'est l'autoréférence processuelle.

L'autoréférence n'est pas l'égocentrisme. Elle exprime avant tout le fait que toute connaissance passe par un sujet existant et «computant" (selon l'expression d'Edgar Morin), au sens de collectant et traitant de l'information. Elle «n'est pas, la propriété substantielle, organique ou formelle, qui permet à un système (vivant en l'occurrence) de se désigner lui-même ; c'est l'aptitude à s'auto-computer à la fois comme objet et sujet. L'autoréférence ne se résume nullement dans l'acte de se référer à soi. Elle est la capacité de se référer à soi tout en se référant à ce qui n'est pas soi. [...]. Elle lie la référence à soi et la référence à ce qui est autre : l'environnement et les choses de l'environnement. Le caractère auto-exoréférent du computo pose et éclaire à la source le problème des possibilités et limites de la connaissance objective pour un être vivant » (Morin, 1986, p. 45).

Pour introduire le troisième invariant, $l^{\prime}$ indétermination, écoutons Werner Heisenberg (1998, p. 258), physicien allemand, Prix Nobel de physique en 1932, qui propose le parallèle avec la vue: "Pour prendre une comparaison, l'œil humain n'est capable d'une observation très nette que sur une petite zone de la rétine et c'est pourquoi il s'oriente toujours inconsciemment de telle sorte que la partie la plus importante de l'image se trouve à cet endroit. La pensée humaine fait de même : elle saisit chaque fois un petit contenu partiel déterminé qu'elle amène à la pleine clarté de la conscience, tandis que le contenu résiduel de ce qui est pensé n'apparaît que dans une pénombre obscure. De cet "essentiel" d'une pensée, le langage peut donner une représentation. » Entendons-le encore un peu plus loin à propos de l'observation: "Cette intervention de l'observation entraîne en outre la conséquence que les propriétés du système ne peuvent pas être toutes objectivées simultanément. Les propriétés particulières se trouvent au contraire souvent dans des rapports "complémentaires" les unes avec les autres. On entend par là que l'objectivation, c'est-à-dire l'établissement par l'observation d'une propriété déterminée, exclut la connaissance de certaines autres propriétés déterminées. L'état est modifié par l'observation d'une propriété déterminée du système de telle sorte qu'une connaissance acquise, par exemple au cours d'observations antérieures, au sujet de la valeur ou de la valeur probable d'une autre propriété est par là même définitivement perdue » (Heisenberg, 1998, p. 302).

$C^{\prime}$ est donc en mettant en évidence le caractère parcellaire de la connaissance que W. Heisenberg nous met sur la voie du troisième invariant, l'indétermination. Notons que c'est bien d'indétermination, et non pas d'incertitude, qu'il parle. Il s'agit bien, en effet, pour lui de désigner l'état de tout système avant que l'observateur ne lui prête des propriétés ${ }^{11}$ et non pas de faire état du caractère incertain d'une expérimentation et de l'ignorance subjective de l'expérimentateur. L'invariant d'indétermination ne signifie pas un choix au sens autoréférent, mais le recours inéluctable à une précision sélective. Il se présente selon nous également sous trois formes. Nous le comprenons comme l'exercice à la fois d'une sélection, $d^{\prime}$ une circonscription et/ou d'une focalisation. La sélection exprime une mise en priorité en fonction d'un objet d'attention. C'est l'indétermination structurelle qui est affaire de cadrage. La circonscription est situationnelle au sens où il va s'agir de considérer une situation qui spécifie les attributs du contexte. La focalisation suggère la recherche d'une netteté qui dépend, selon la distance, d'un réglage de la focale de l'observateur ou du dispositif d'observation. C'est l'indétermination processuelle. Délimiter, mettre en situation et rendre net constituent trois facettes liées et nécessaires en vue d'attribuer des propriétés aux objets que l'on perçoit. L'indétermination prend fin dès que l'observateur prête des propriétés à l'objet étudié.

Au terme de cette présentation des invariants, il nous faut dire qu'ils ne sont nullement indépendants les uns

\footnotetext{
11 Catherine Chevalley, dans son introduction à Philosophie : le manuscrit de 1942 (Heisenberg, 1998), le rappelle avec force: "L'indétermination des "relations de Heisenberg" est une indétermination qui n'est en rien une incertitude ou un vague conceptuel » (1998, p. 164).
} 
des autres (on aura noté, chez Heisenberg particulièrement, à quel point les notions d'incomplétude et d'autoréférence sont mêlées à la définition de l'indétermination). Ils sont dans un état d'interdépendance inéluctable. En physique, on parlerait d'une « coextensivité », associée à l'idée d'un champ potentiellement présent partout, bien qu'invisible, par exemple, le champ gravitationnel. Parler de coextensivité entre les invariants introduit l'idée d'une existence singulière de chacun d'eux sans aucune prépondérance de l'un d'entre eux sur les autres. Ils sont à la fois singuliers et coextensifs, combinaison fondamentale pour fonder la nature relationnelle - on pourrait dire la viabilité relationnelle - de la méthode proposée.

\section{La nature relationnelle de la connaissance}

De ce qui précède, il découle que la connaissance que l'on se construit du monde naît des relations entre les invariants. Dans chaque invariant, on retrouve un reflet ou une influence des deux autres. L'incomplétude, l'autoréférence et l'indétermination, qui constituent les entrées d'une possible connaissance ternaire, engagent, comme nous venons de le dire, une inséparabilité causale dans les sphères isomorphes des idées et de la réalité perçue. On peut parler de «concept-phénomène » pour exprimer la façon dont nous considérons la réalité phénoménologique à laquelle nous avons accès par nos perceptions et nos représentations: la réalité s'élabore en pensée par les mots qui saisissent la réalité et construisent la pensée (Heisenberg, 1998, p. 259-260). Ce même Heisenberg emploie le terme de "connexion" pour exprimer l'idée que ce ne sont pas les objets qui sont constitutifs de la réalité mais l'enchaînement des relations entre eux ${ }^{12}$. Et cela s'applique aussi bien à l'ordre matériel même qu'à la façon de le penser. Cette idée se retrouve au fondement de l'approche ternaire que nous proposons.

L'interprétation recherchée est donc de nature relationnelle aux plans des concepts-phénomènes que l'analyste retient. Les termes ne sont désignés qu'en lien les uns avec les autres, dans une combinatoire interprétative. On mesure pleinement l'importance du langage, des saisies langagières multiples qui mettent les concepts et les vocables en relation et cela a été bien compris $^{13}$ (Heisenberg, 1998 ; Morin, 1986 ; Wunenburger,

12 Ce qu'il y a de permanent dans la réalité serait alors à rechercher du côté des lois qui fondent les relations par lesquelles on la saisit : «Ce qui est permanent à travers la variation des phénomènes n'est pas la chose matérielle, c'est la loi. » (Heisenberg, 1998, p. 273).

13 Le concept de résilience, compris comme englobant ou transverse, envisagé avec de nombreux autres termes qui s'y associent ou le précisent, constitue un bon exemple de ces constructions langagières (Hervé et Rivière, 2014).

\section{Encadré 2. L'antagonisme non symétrique}

S. Lupasco parle $\mathrm{d}^{\prime}$ «antagonisme » quand on se trouve face à deux agents dont les influences s'opposent tout en étant nécessaires l'une à l'autre pour penser le dynamisme du phénomène que recouvre l'antagonisme. Cette opposition se comprend comme une tension énergétique (pensons, par exemple, au jeu de deux muscles qui se contrarient, mais qui sont nécessaires l'un à l'autre pour maintenir une dynamique). L'antagonisme est dit non symétrique, ce qui est le cas courant, quand la contradiction entre les agents qu'il oppose n'est pas frontale, mais joue seulement sur certaines facettes, sur la base d'intensités et de registres d'opposition potentiellement différents. La nonsymétrie fonde la possibilité d'une dynamique et d'un dépassement.

1990). Mais comment envisager méthodologiquement cette lecture relationnelle?

Nombre d'auteurs ont mis en évidence le fait que, dans un monde d'interconnexions, isoler un phénomène ou un événement, effectuer une expérience et donner une interprétation engagent un découpage de la réalité qui produit des contradictions dans ce qui est observé ou, dit autrement, qui explique pour une part essentielle les contradictions que l'on y repère. Cette difficulté, bien identifiée $^{14}$, suggère qu'un des moyens de dépasser le simple repérage de ces contradictions est de modifier le référentiel utilisé pour saisir cette réalité. Dans ce cadre, l'interprétation " énergétique » de l'antagonisme suggérée par Stéphane Lupasco (1987) s'affirme comme fondamentale ( $c f$. Encadré 2). La logique de l'énergie qu'il défend est de portée universelle. Elle vaut aussi bien pour les énoncés mentaux que pour les phénomènes physiques que les énoncés mentaux désignent. Elle a d'ailleurs largement nourri la compréhension de ce que pourrait être une pensée dialogique ou contradictoire susceptible de saisir le dynamisme des phénomènes, comme nous l'avons évoqué plus haut. Mais une question se pose : comment, face à deux phénomènes contradictoires (saisis par des concepts), conserver l'antagonisme non symétrique qu'ils représentent ? La quête de ce que nous convenons d'appeler les «tiers incluants » constitue la réponse.

Par le qualificatif d'incluant est signifié le fait que l'élément tiers, concept et/ou phénomène, inclut les agents de l'antagonisme. Il maintient possible (compatible) en son sein, par englobement, l'expression de logiques contraires, donc d'un antagonisme et d'une

14 Cf. notamment M. Mugur-Schächter (2006), op. cit., ou bien encore B. Nicolescu dans la préface de l'ouvrage de S. Lupasco (1987). 
tension énergétique. Exprimé différemment, le tiers incluant recouvre la possibilité du jeu oscillatoire de l'actualisation et de la potentialisation proposé par S. Lupasco (1987). Le tiers incluant s'avère un acteur de l'antagonisme maintenu quand le tiers inclus signifie une extériorité $^{15}$ eu égard aux termes opposés. On prend alors conscience de l'ambiguité qu'il y a à opposer le tiers inclus au tiers exclu aristotélicien. Le tiers non exclu, nous le comprenons comme le tiers incluant les termes de l'antagonisme et non comme le tiers inclus. Confortons ces idées par un exemple portant sur les pièces mécaniques d'une voiture, comme le moteur et le système de freinage, deux dispositifs potentiellement antagonistes (le moteur imprime un déplacement et le frein l'empêche). Tant que la voiture est à l'arrêt, ces deux dispositifs sont dans un simple rapport de complémentarité inerte englobé dans l'objet "voiture ». C'est si l'on envisage la conduite du véhicule qu'une relation causale (dit autrement: une continuité ${ }^{16}$ ) s'établit entre eux. Une unité dynamique s'instaure alors dans un processus qui exige à la fois possibilité de déplacement et possibilité d'immobilisation. Le jeu du tiers incluant repose sur l'exploitation d'une continuité, donc d'une relation causale entre des termes séparés, qu'établit l'analyste par un changement de référentiel. Parce qu'il s'agit de continuité, le tiers incluant n'est donc pas un médiateur ou un conciliateur ou encore un révélateur de complémentarité.

Ainsi, la lecture relationnelle annoncée prend appui sur les trois couples de relations binaires entre les invariants. Une première caractéristique $\mathrm{du}$ complexe par rapport au compliqué serait le passage du binaire au ternaire tout en incorporant le premier dans l'analyse relationnelle. Une seconde caractéristique, corollaire de la première, serait l'essor d'une circulation. Nous allons chercher à faire vivre cette lecture relationnelle que nous qualifions de "trialectique » pour saisir le phénomène de la perception.

15 Qui est bien souvent elle-même signifiée en évoquant l'idée d'un méta niveau ou d'une méta position. Mais cette compréhension sous forme d'association d'idées est une difficulté car on ne saurait dire que le tiers incluant occupe une position méta ou qu'il suffise de dire que l'observateur se pose en position méta pour qu'existe la possibilité du tiers incluant.

16 La continuité est (paradoxalement) connexe de la séparation. Deux interlocuteurs séparés l'un de l'autre, présentant de très nombreuses différences, ne peuvent communiquer que grâce à la continuité apportée par le milieu contextuel en termes d'espace-temps physique et culturel communs. Ce milieu permet la séparation identitaire corporelle et mentale, mais en même temps la continuité requise pour l'échange, jusqu'à l'air qui assure la transmission du son ou bien encore la circulation des photons qui permet la vision. Continuité et séparation vont ensemble comme d'ailleurs discontinuité et non-séparabilité dans l'univers quantique.

\section{La perception au fondement de la posture épistémologique}

Le phénomène de la perception constitue la première mise en intelligibilité du processus d'observation consciente. Son analyse prend appui sur la reconnaissance du caractère partiel, partial et parcellaire de toute perception et partant de toute connaissance.

\section{L'analyse structurelle de la perception : processus d'observation consciente et attracteurs}

La compréhension du phénomène de la perception qui fonde l'accès à la réalité suppose un processus d'observation consciente qui est significativement placé au centre du schéma de la perception (Fig.). L'exercice de la pensée à réaliser est de concevoir ce processus associé à une conscience de la conscience, autrement dit à une faculté réflexive. Nous partons alors en quête des propriétés du phénomène de la perception déclenchées par le processus d'observation consciente. Il faut insister sur le fait que c'est bien le phénomène ou processus de perception qui est analysé et non ce qui est perçu.

Le schéma ternaire distingue trois sphères correspondant aux invariants fondamentaux (incomplétude, autoréférence, indétermination) et aux attracteurs du thème étudié (partiel, partial, parcellaire), ici la perception. Les sphères indiquent les agents d'influence, au nombre de deux par sphère. Les chevauchements traduisent qu'il y a coextensivité ou inséparabilité causale et donnent à voir les influences réciproques des invariants les uns sur les autres. Les trois fuseaux de chevauchement, correspondant aux trois couples de "frottements » binaires, indiquent les antagonismes identifiés et les tiers incluants. Les sphères de l'incomplétude et de l'indétermination sont colorées de couleur froide, respectivement en vert et en bleu, pour traduire qu'il ne s'agit pas de jugements ou de choix mais bien de facettes "froides » des phénomènes, d'aspects inéluctablement associés à $l^{\prime}$ incomplétude et à l'indétermination ${ }^{17}$. Cette distinction n'exclut pas l'influence de l'autoréférence, associée, quant à elle, à une couleur chaude car toujours expression d'un choix au sens d'une volition, d'une intention ou d'un point de vue. Enfin, la quête et l'identification des mots empruntent au référentiel de l'optique (ou de la vue), ce qui permet, pensons-nous, une compréhension facilitée des phénomènes physiques et mentaux en jeu.

À l'invariant de l'incomplétude correspond l'attracteur de la perception partielle : le partiel. Nous avions précédemment associé à cet invariant de l'incomplétude trois formes de compréhension que nous retrouvons ici dans le phénomène premier de la perception : l'existence

\footnotetext{
17 Ces couleurs apparaissent dans la version numérique (PDF) de l'article accessible sur le site de l'éditeur (www.nss-journal.org) ou sur la plateforme Cairn (www.Cairn.info)
} 


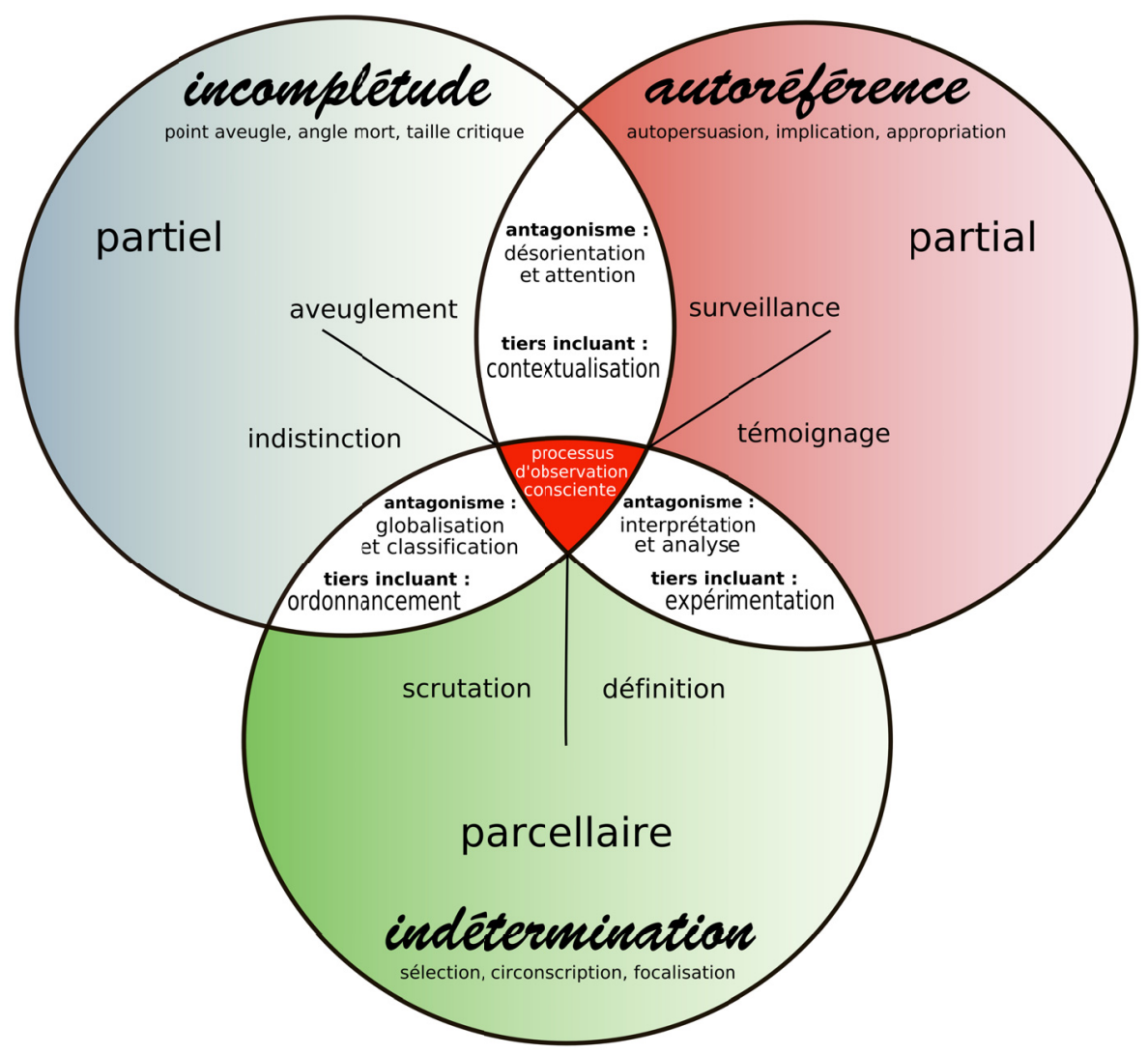

Fig. Le schéma ternaire de la perception.

inéluctable d'un point aveugle dès lors que l'on existe ou que l'on fait partie de la réalité observée, le caractère inévitable d'un angle mort en lien avec une localisation dans l'espace-temps, l'expression incontournable d'une taille critique entendue comme un maximum possible de ce qui est perçu. À l'invariant de l'autoréférence correspond l'attracteur de la perception partiale : le partial. Il manifeste la subjectivité de l'interprétation inhérente à toute perception. C'est la reconnaissance du point de vue associé aux phénomènes d'autopersuasion, d'appropriation et d'implication. À l'invariant de l'indétermination correspond l'attracteur de la perception parcellaire : le parcellaire. Il nous dit que toute perception peut selon les cas, et simultanément ou non, être considérée comme une sélection, une circonscription ou une focalisation.

Nous voici donc avec trois dérivées des invariants pour le thème de la perception, soit les trois attracteurs du partiel, du partial et du parcellaire $\mathrm{qu}^{\prime}$ il va falloir mobiliser conjointement dans l'analyse relationnelle.

\section{L'analyse relationnelle de la perception : agents d'influence, antagonismes et tiers incluants}

L'analyse relationnelle s'engage sur la base de l'identification des relations qui se nouent entre les invariants et les attracteurs (voir le schéma de la perception ). Il s'agit donc de partir en quête des agents d'influence ou d'interaction inscrits dans les sphères du schéma de la perception.

Pour l'agent de l'incomplétude sur le parcellaire (la perception parcellaire), nous proposons l'indistinction. Celle-ci manifeste que la quête du parcellaire poussée aussi loin que possible butera sur l'impossibilité de distinguer l'objet ou le phénomène observé en raison de phénomènes de point aveugle, d'angle mort ou de taille critique. L'incomplétude agit sur le partial par le manque inéluctable qu'elle introduit et qui rend la perception partiale incomplète. Nous considérons que c'est l'aveuglement, compris non comme le refus de voir, mais comme l'impossibilité d'une vision totale qu'entraînent le point aveugle, l'angle mort ou la taille critique.

Pour l'agent de l'autoréférence (et les phénomènes d'autopersuasion, d'appropriation et d'implication qu'elle recouvre) sur la perception partielle, nous proposons la surveillance, c'est-à-dire l'intention ou l'intentionnalité qui se manifeste par une vigilance d'observation sur la réalité perçue. Nous identifions l'agent de l'autoréférence sur le parcellaire comme le témoignage, compris comme une attention partiale, une attitude qui rend compte de manière subjective des propriétés d'un phénomène ou d'un objet observé.

L'indétermination agit sur le partial à travers la définition (au sens de l'optique) qui précise la perception 


\section{Commentaire synthétique}

Les dérivés des invariants de l'incomplétude, de l'autoréférence et de l'indétermination sont respectivement les attracteurs du partiel, du partial et du parcellaire. Ces trois attracteurs du thème de la perception sont en relation à travers l'influence qu'exercent les invariants entre eux. Les agents de l'incomplétude sont l'indistinction sur le parcellaire et l'aveuglement sur le partial. Les agents de l'autoréférence sont la surveillance sur le partiel et le témoignage sur le parcellaire. Les agents de l'indétermination sont la définition sur le partial et la scrutation sur le partiel. L'exercice qui consiste à renommer les agents pour mieux identifier l'antagonisme qui se joue entre eux conduit à identifier les tiers incluants de la contextualisation, de l'expérimentation et de l'ordonnancement.

partiale (un certain degré de précision) en vertu du fait que sont à l'œuvre des phénomènes de sélection, de circonscription et de focalisation dans un champ donné de perception. Nous considérons que la scrutation, agent de l'indétermination dans le partiel, permet la précision de sélectivité.

Ainsi, chaque invariant participe des deux autres dans la relation de coprésence qui les lie. Ils représentent trois polarités interprétatives inséparables que nous cherchons à qualifier par l'identification des agents d'influence. Ceux-ci, nous venons de le voir dans le cadre du schéma ternaire de la perception, décrivent des influences entre un invariant et l'attracteur du thème dans les deux autres sphères. Il s'agit d'une interprétation « libre » dans le cadre d'une règle d'exécution toutefois rigoureusement posée. Cette appréciation que l'on pourrait qualifier d'arbitraire, W. Heisenberg (1998, p. 259) la précise utilement : «Dans la région de la pensée "statique", on explique, dans la mesure où le but véritable de cette forme de pensée est avant tout la clarté ; dans la région de la pensée "dynamique", on interprète, car ce qu' on recherche ici, ce sont des relations infiniment variées, avec d'autres régions de la réalité que nous puissions interpréter ». Ainsi, l'identification des invariants constitue une étape de clarification référentielle qui permet ensuite une interprétation dynamique des relations « infiniment variées » que ces invariants entretiennent et font naître selon diverses lectures possibles de la réalité.

Il convient maintenant d'envisager les contradictions ou antagonismes et l'identification des tiers incluants qui permettent de les conserver au plan explicatif. Nous avons précédemment identifié trois paires d'agents au sein des sphères de chaque invariant. Ceux-ci présentent des antagonismes qui apparaissent de part et d'autre des fuseaux.

Entre le partiel et le partial, l'aveuglement et la surveillance constituent deux états de toute évidence contraires. Entre le partial et le parcellaire, on trouve le témoignage et la focalisation. Ces deux actions agissent dans deux registres opposés : 1'un est une manière de faire un récit général et subjectif comme témoin direct d'un événement survenu, et l'autre vise à inspecter une situation d'une manière détaillée. Entre le parcellaire et le partiel figurent l'indistinction et la scrutation. Ce sont aussi deux manières opposées d'exercer le regard, car l'indistinction représente une vision englobante sans préférence d'attention particulière.

Le schéma reprend les agents renommés que nous avons retenus : aveuglement et surveillance deviennent désorientation et attention; indistinction et scrutation deviennent globalisation et classification; témoignage et définition deviennent interprétation et analyse. La reformulation cherche à (re)monter dans le niveau de conceptualisation en passant du spécifique au plus englobant afin de trouver la classe d'objets supérieure, le dénominateur commun. Par exemple, un témoignage est englobé dans l'interprétation qui est son phénomène fondateur. Ayant franchi cette étape, nous cherchons le tiers incluant qui va signifier cette énergie des contraires à un niveau de réalité englobant.

Pour l'antagonisme entre désorientation et attention, le tiers incluant choisi est contextualisation. Désorientation et attention se montrent bien antagonistes car il n'est pas possible d'être attentif dans la désorientation, c'est-àdire sans direction privilégiée à prendre en considération. La désorientation est une absence de repères connus de l'observateur, ce qui rend impossible l'attention mais, dans le même mouvement, la désorientation ne peut se dire que parce que l'attention a permis de la constater. La contextualisation doit signifier la problématique au sein de laquelle s'effectue une telle " ronde » entre désorientation et attention.

Expérimentation est le tiers incluant retenu pour interprétation et analyse. L'interprétation relève de la subjectivité ; l'analyse suppose un relevé d'informations dans un domaine bien défini à partir duquel on pourra dire qu'il y a objectivité contextuelle. L'expérimentation semble bien la capacité proprement humaine qui, au fil des siècles, a permis les progrès de la connaissance.

Enfin, ordonnancement est retenu comme tiers incluant pour globalisation et classification. On ne peut classer que ce qui apparaît comme des éléments distincts et différenciés, et c'est parce qu'il y a ordonnancement qu'une certaine globalisation peut être effective. Signalons aussi que le tiers incluant que nous cherchons appartient aux deux sphères «froides » de l'indétermination et de l'incomplétude par opposition à la sphère « chaude » de l'autoréférence. 
Tableau 1. De la perception vers le référentiel généralisé.

\begin{tabular}{|c|c|c|}
\hline Attracteur & Attribut associé & Référentiel \\
\hline partiel & exception & domaine de validité \\
\hline partial & conception & champ d'intelligibilité \\
\hline parcellaire & acception & degré de résolution \\
\hline
\end{tabular}

Ainsi, l'analyse trialectique de la perception nous indique que toute perception inéluctablement partielle, partiale et parcellaire met en jeu une contextualisation, une expérimentation et un ordonnancement. Ces trois "capacités » inhérentes au fait de percevoir peuvent aisément être associées à la capacité d'appréhender le monde, de le saisir et d'être "saisi » si l'on veut bien jouer sur les deux compréhensions du terme « appréhender ». Une exégèse plus approfondie de la cartographie obtenue mériterait d'être menée mais nous souhaitons privilégier un prolongement: la perception, comprise comme partielle, partiale et parcellaire, nous guide vers ce qu'elle implique, à savoir le référentiel généralisé sur le plan des connaissances et la réalité phénoménologique sur le plan de l'existence.

\section{De la perception vers le référentiel généralisé et la réalité phénoménologique}

La perception, fruit du processus d'observation consciente, constitue l'entrée première dans l'existence physique et idéelle au monde. Elle nous guide, au plan méthodologique et conceptuel, vers une double filiation : la connaissance et la réalité phénoménologique. La première voie est celle des attracteurs de la perception, à savoir le partiel, le partial et le parcellaire qui nous guident vers la connaissance et ses attributs. La seconde passe par les tiers incluants de la perception, à savoir la contextualisation, l'expérimentation et l'ordonnancement qui nous désignent la réalité phénoménologique.

Abordons en premier lieu le lien qui s'établit entre la perception et le référentiel généralisé dans lequel s'inscrit toute connaissance (Tab. 1). Nous introduisons une colonne nouvelle, " attribut associé », faisant figurer un concept intermédiaire entre l'attracteur et le référentiel pour une meilleure compréhension du raisonnement.

La perception partielle (le «partiel»), qui émane de l'incomplétude, peut se traduire par l'exception en ce sens que cet attracteur représente la prise en compte la plus complète possible d'une entité ou d'un phénomène (mais toujours à l'exception d'une partie du phénomène, du fait des limites liées au point aveugle, à l'angle mort ou à la taille critique). Le dictionnaire définit l'exception comme signifiant "retirer", "mettre à part ", "soustraire à la règle commune ». Conséquence du partiel, l'exception pose toujours la question de la validité par rapport à une norme ou une régularité. L'un des axes du référentiel sera donc le domaine de validité des connaissances provoqué par l'invariant de l'incomplétude.

En relation à l'autoréférence, le «partial » exprime l'influence de la conception personnelle, au sens d'intentionnelle ou voulue de l'observateur ou analyste. Le dictionnaire définit la conception comme signifiant une « opération de l'esprit appliquée à un objet », ou encore un "saisi imaginé ». L'un des axes du référentiel sera donc le champ d'intelligibilité déclenché par l'invariant de l'autoréférence. Si le cercle de l'incomplétude est froid et élimine l'invalide, celui de l'autoréférence est chaud et va décider de ce qui est pertinent en créant cette pertinence par le regard propre de l'observateur, du fait des phénomènes d'autopersuasion, d'appropriation et d'implication, non sans inflexion bien sûr de la part des deux autres invariants.

En relation à l'indétermination, le "parcellaire » est une prise en compte la plus précise possible d'une entité mais toujours en fonction d'une sélection, d'une circonscription ou d'une focalisation. C'est une acception de la réalité qui s'impose à l'observateur par le jeu des trois phénomènes que nous venons d'évoquer et que l'on peut comprendre comme un choix de résolution. L'un des axes du référentiel sera ainsi le degré de résolution déclenché par l'invariant de l'indétermination. Le dictionnaire le définit comme « la distance minimale qui doit exister entre deux points contigus pour qu'ils soient correctement distingués ». Le degré de résolution indique que si l'on est clair sur le domaine ainsi que sur le champ, l'absence de précision sur la région choisie empêchera tout traitement d'information ou toute action concluante, dès lors que les contours de l'objet d'attention n'auront pas la netteté requise.

Ainsi, la perception qui a pour attracteurs le partiel, le partial et le parcellaire, induit une exception qui engage l'identification d'un domaine de validité, une conception qui engage un champ d'intelligibilité et une acception qui implique un degré de résolution. Ces opérations sont fondamentales et se retrouvent dans toute relation de connaissance au monde, à notre environnement ou à nous-mêmes. L'incomplétude, créatrice du domaine, manifeste l'impossibilité d'embrasser tous les champs à la fois comme de prendre en compte tous les degrés. L'autoréférentialité des champs ouverts à 
Tableau 2. De la perception vers la réalité phénoménologique.

\begin{tabular}{|c|c|c|}
\hline Attracteurs antagonistes & Tiers incluant & Réalité phénoménologique \\
\hline partiel/partial & contextualisation & relativité \\
\hline partial/parcellaire & expérimentation & humain \\
\hline parcellaire/partiel & ordonnancement & organisation \\
\hline
\end{tabular}

l'intelligibilité va introduire du subjectif dans la «physicalité froide » de l'incomplétude et soustraire à l'indétermination la résolution de ce même choix par le bon vouloir qu'elle exprime. Et bien sûr, l'indétermination imprégnée des deux autres ne les en contraindra pas moins à spécifier le domaine tout comme à limiter le pouvoir de l'autoréférence par l'impératif de la précision sélective. Domaine de validité, champ d'intelligibilité et degré de résolution représentent trois éléments constitutifs d'un référentiel qu'on peut qualifier de généralisé dans la sphère des connaissances.

Nous venons de privilégier la question de la connaissance qui advient avec la perception, mais la question de l'existence au monde et à la réalité co-occurrente n'est pas loin. Les tiers incluants de la perception, à savoir contextualisation, expérimentation et ordonnancement, nous offrent la voie d'accès que nous empruntons maintenant (Tab. 2).

Le tiers incluant de la contextualisation, ici spatiotemporelle, au niveau duquel se résout l'antagonisme entre désorientation et attention, fait appel fondamentalement à une posture relativiste au sens d'Einstein. L'idée d'une position absolue et calculable pour un objet n'a plus de sens dans l'ordre cosmique : toute chose, pour être située, nécessite un référentiel et tout référentiel engage un point de vue, une perception autoréférente. Ainsi la contextualisation, dans le sens riche dans lequel nous la comprenons, à savoir un tiers incluant empruntant à la fois aux influences de l'incomplétude et de l'autoréférence pour ce qui est de la perception, désigne la relativité générale ${ }^{18}$ comme première sphère de la réalité phénoménologique.

L'expérimentation, tiers incluant au niveau duquel se résout l'antagonisme entre le partial et le parcellaire, identifié comme couple interprétation-analyse, dépend d'un expérimentateur. Elle nous désigne l'humain ${ }^{19}$, et l'on comprend que toute expérimentation qui engage un humain expérimentateur sur la sphère terrestre engage

\footnotetext{
18 Les trois attracteurs du schéma ternaire de la relativité générale sont l'espace-temps (incomplétude), l'énergie (autoréférence), la matière (indétermination).

19 Les trois attracteurs de l'humain suggérés directement par E. Morin sont l'espèce (incomplétude), l'individu (autoréférence), la société (indétermination).
}

aussi une organisation de la réalité en même temps que le regard s'organise et organise cette réalité ${ }^{20}$.

L'ordonnancement, tiers incluant au niveau duquel se résout l'antagonisme entre le parcellaire et le partiel, identifié comme couple globalisation-classification, pointe la nécessité d'identifier un niveau organisationnel observable de la physicalité : c'est l'organisation dans ses multiples systèmes emboîtés que le monde physique recouvre qui est ainsi désignée. Nous comprenons l'ordonnancement comme une différenciation, une structuration qui dans son acception la plus large implique un tri, des priorités, des séparations, des réductions, des mises en hiérarchie ou en préséance, des intégrations diverses de nature systémique. L'organisation ${ }^{21}$ sera ainsi une facette de la réalité phénoménologique, bien sûr inséparable des deux autres que sont la relativité générale et l'humain.

Concluons, à ce stade, le cheminement que nous venons d'emprunter à partir de la perception. Comme nous avons pu le dire, l'ensemble du schéma de la perception se construit sur la base de la reconnaissance du processus d'observation consciente, phénomène désincarné et possible en tout point de l'univers. Ce processus fait écho à la Limite (Encadré 3) à laquelle il permet de se confronter à travers le référentiel généralisé et la réalité phénoménologique. Ces derniers naissent avec la perception qui naît aussi avec eux.

La perception représente un dispositif d'accès ou de médiation vers le référentiel et la réalité. Quel que soit le système percevant, le processus d'observation consciente engage une perception partielle, partiale et parcellaire qui constitue une médiation fondamentale vers le référentiel généralisé et la réalité phénoménologique. Le référentiel au plan des connaissances nous instruit sur les considérations épistémologiques et méthodologiques imbriquées qui naissent de l'exercice réflexif d'une perception consciente de percevoir. La relativité, l'humain et l'organisation nous suggèrent que, de la perception, naît une nécessaire compréhension transdisciplinaire de la réalité qui imbrique ces trois facettes.

\footnotetext{
20 Au sens où l'expérimentation est associée à une réflexivité entendue comme conscience de la conscience.

21 Les trois attracteurs de l'organisation sont l'écologie (incomplétude), la téléologie (autoréférence), l'ingénierie (indétermination).
} 


\section{Encadré 3. La Limite}

La Limite, au singulier et avec une majuscule, exprime $l^{\prime}$ « inconnaissabilité » à laquelle confronte le fait même d'exister. Mais la Limite ainsi comprise comme finitude radicale de l'existence et de la connaissance manifeste aussi l'existence et la connaissance qu'elle rend possible. Dans la lecture ternaire proposée, la confrontation à la Limite se comprend à travers le jeu des invariants de l'incomplétude, de l'autoréférence et de l'indétermination qui rend possible l'accès à la connaissance. La Limite est à penser en lien avec le concept de courbure par lequel on saisit l'espacetemps. Ce lien établit une compréhension de la Limite qui s'éloigne des acceptions linéaires, communément répandues, d'une limite toujours repoussée.

La perspective d'objectivité n'en est pas pour autant exclue, au sens de la possibilité et de la pertinence d'un processus d'objectivation rigoureux, fondé notamment sur la pleine lucidité d'un domaine de validité, d'un champ d'intelligibilité, d'un degré de résolution. L'objectivation est toujours contextuelle. Cette position n'exclut nullement que l'on reconnaisse des lois (une légalité plus qu'une causalité22) dont on peut dire que leur expression se manifeste très concrètement, contextuellement, comme les lois de Newton sur la gravitation dont on sait la portée dans le contexte des affaires terrestres mais aussi les limites au-delà avec Einstein. La physicalité n'est bien sûr nullement exclue de la réalité, pas plus que la place de l'expérimentation ou de l'expérience pour valider les lois posées comme hypothèses. La nier correspondrait à une compréhension pauvre, relativiste et psychologisante du principe de réalité phénoménologique ou perçue. À la réalité telle que nous la percevons correspond une physicalité.

\section{Conclusion}

Cette contribution n'est pas à proprement parler philosophique. Elle repose toutefois sur une ingénierie qui engage une compréhension de notre rapport physique et idéel au monde qui, elle, se trouve bien au fondement de la réflexion philosophique. Elle ne vise pas non plus un domaine scientifique particulier, pas plus qu'elle ne part d'un ancrage dans une discipline singulière. En ce sens,

22 J. Piaget (1970, p. 101-103) retient que la légalité appartient au domaine des observables, tandis que la causalité est inobservable et seulement déduite, d'où, paradoxalement, la méfiance traditionnelle de l'empirisme puis du positivisme à son égard. Lors de l'action d'un mobile sur un autre, on perçoit que quelque chose a " passé » mais on ne voit rien " passer ». elle serait transdisciplinaire. Nous avons ainsi le sentiment de contribuer à la quête de cette «brique transdisciplinaire » que le groupe de recherche Academos (2006) identifie au carrefour des philosophies et des sciences et qui toucherait au cœur de la pluri ou de l'interdisciplinarité $^{23}$ (A.-F. Schmid in Academos, 2006, p. 54-55). De ce point de vue, nous pouvons témoigner de l'intérêt d'être nourris de réflexions issues de disciplines différentes, dans notre cas des sciences sociales (sociologie, économie...) et de la physique, physique quantique notamment. Nous pensons que la perspective méthodologique proposée peut contribuer à la réflexion pluri, inter ou transdisciplinaire, en menant une investigation sur les conditions premières de formation de toute connaissance ; il est déjà acquis pour nous qu'elle s'en nourrit.

Si les véritables problèmes de l'interdisciplinarité résultent de la diversité des modes d'acquisition des connaissances et de construction des «faits » (Mathieu et Jollivet, 2013), nous espérons avoir apporté une pierre à l'édifice interdisciplinaire en mettant au jour les limitations en même temps que les conditions de toute connaissance.

$\mathrm{Au}$ terme du cheminement d'ensemble et des questions qu'il n'a pas manqué de poser à ses auteurs mais aussi sans doute aux lecteurs, cet article conclut sur un enseignement que l'on peut énoncer en quelques mots : nous construisons le monde et la connaissance du monde sur $1^{\prime}$ « inconnaissabilité » radicale qui accompagne notre propre présence connaissante. D'où, peut-être, l'impression d'un formalisme excessif, mais il s'agit bien d'une perspective méthodologique à faire vivre et à partager dans la pratique de l'interdisciplinarité. Insistons encore sur le fait que ce qui est proposé doit être compris comme une réflexion en cours, non figée dans une quelconque forme d'aboutissement qui deviendrait rigidité. Mais cette construction suppose un accès auquel nous avons essayé ici de contribuer sur un mode scientifique, c'est-à-dire ouvert à la critique sur la base d'une explicitation de ses fondements et de ses raisonnements.

L'inconnaissabilité qui accompagne notre naissance à nous-mêmes, à la conscience de notre conscience, est au fondement de la possibilité de connaître, de la possibilité de penser notre pensée: "How can thought think of itself? » ${ }^{24}$, se demandait en $1872^{25}$ C. S. Peirce, l'un des pères du pragmatisme, en constatant immédiatement

\footnotetext{
23 Nous retenons que la multi ou pluridisciplinarité consiste à aborder un objet d'étude en associant les regards disciplinaires au risque du morcellement de l'objet d'étude lui-même, quand l'interdisciplinarité recherche la collaboration disciplinaire en travaillant à des saisies conceptuelles et langagières partagées.

24 «La pensée peut-elle penser la pensée?».

25 MS 192 (Robin 395): Writings 3, 10-11, Summer-Fall 1872, http:/ / www.iupui.edu/ arisbe/menu/library/bycsp/logic/ ms192.htm
} 
qu'il ne pouvait y répondre que par les moyens de la pensée : "The question is, what is thought, and the question can only be answered by means of thought. (...) I have only to act as though my thought were an external object. ». Il devait penser sa pensée comme un objet extérieur à lui-même. À cette interface, la méthode trialectique trouve sa place en tant qu'ingénierie du processus de structuration de la pensée appliquée à la pensée et l'univers phénoménal.

Le phénomène de la perception, dont on sait l'importance pour le courant phénoménologique, pose deux questions liées : celle de notre capacité perceptrice ellemême, qui fonde notre rapport à la réalité, celle de notre capacité à exercer notre raison ou à penser le phénomène de la perception lui-même. C'est à bien des égards ces deux questions mêlées que l'ingénierie proposée prend «à bras-le-corps » dans la cartographie ternaire relationnelle qu'elle avance pour tenter d'élucider ce qui se joue dans notre rapport conscient au monde. Ce dispositif vise à permettre d'exercer notre raison consciente d'ellemême sur la perception qui constitue notre accès premier à la connaissance.

La transdisciplinarité dont il est souvent dit qu'elle recouvre ce qui est au-delà de toute discipline et ce qui traverse toutes les disciplines possibles engage logiquement nos manières de concevoir le monde sans découpage disciplinaire a priori. Si l'on conçoit le rapport au monde physique (physique, biologique et social) et idéel à travers les trois invariants de l'incomplétude, de l'autoréférence et de l'indétermination, la transdisciplinarité, en tant qu'elle désigne la connaissance, met alors en jeu la perception (partielle, partiale, parcellaire), le référentiel généralisé (domaine de validité, champ d'intelligibilité, degré de résolution), la réalité phénoménologique (relativité générale, humain, organisation). La transdisciplinarité, associée à la complexité attribuée aux phénomènes observés, s'ancre dans le processus de production de connaissances qui la rend accessible.

\section{Références}

Academos, 2006. Philosophies et sciences : pour une «brique transdisciplinaire », Natures Sciences Sociétés, 14, 1, 54-68.

Bréchet, J.-P., 2012. Edgar Morin : la complexité comme défi à la connaissance, in Germain, O. (Ed.), Les grands inspirateurs de la théorie des organisations, Cormelles-le-Royal, EMS.
Descartes, R., 2009 [1 ${ }^{\text {re }}$ éd. : 1637]. Méditations métaphysiques, Paris, Garnier-Flammarion.

De Cues, N., 2013 [texte d'origine daté de 1440]. La docte ignorance, Paris, Flammarion.

Genelot, D., Avenier, M.-J. (Eds), 2012. Agir et penser en complexité avec Jean-Louis Le Moigne. Témoignages de mises en actes, Paris, L'Harmattan.

Gigand, G., 2007. Ingénierie du regard transdisciplinaire. L'événement entre incomplétude, autoréférence et indétermination, Paris, l'Harmattan.

Gigand, G., 2010. Se cultiver en complexité. La trialectique : un outil transdisciplinaire, Lyon, Chronique sociale.

Heisenberg, W., 1998. Philosophie : le manuscrit de 1942, Paris, Seuil.

Hervé, D., Rivière, M., 2014. Résilience, adaptation, changement: l'interdisciplinarité questionnée, Natures Sciences Sociétés, 22, 3, 247-253.

Le Moigne, J.-L., 1990. La modélisation des systèmes complexes, Paris, Dunod.

Le Moigne, J.-L., 2006 [1 ${ }^{\text {re }}$ éd. : 1977]. La théorie du système général. Théorie de la modélisation, Réseau intelligence de la complexité, http://www.mcxapc.org/inserts/ouvrages/ 0609tsgtm.pdf.

Lupasco, S., 1987. Le principe d'antagonisme et la logique de l'énergie, Monaco, Éditions du Rocher.

Mathieu, A., Jollivet, M., 2013. Réflexions pour un bilan: première étape, Journée de l'association NSS-Dialogues (6 décembre 2012), Natures Sciences Sociétés, 21, 3, 355-359.

Morin, E., 1977. La méthode. 1. La nature de la nature, Paris, Seuil.

Morin, E., 1980. La méthode. 2. La vie de la vie, Paris, Seuil.

Morin, E., 1986. La méthode. 3. La connaissance de la connaissance, Paris, Seuil.

Morin, E., 1990. Science avec conscience, Paris, Seuil.

Morin, E., 2009. Logique et contradiction. Communication aux Ateliers sur la contradiction, École nationale supérieure des mines de Saint-Étienne, 19-21 mars, http:/ / www.emse.fr / aslc2009/pdf/Logique \%20et\%20contradiction \%20E\%20 MORIN.pdf.

Mugur-Schächter, M., 2006. Sur le tissage des connaissances, Paris, Hermès/Lavoisier.

Nicolescu, B., 2002 [1 $1^{\text {re }}$ éd. : 1985]. Nous, la particule et le monde, Monaco, Éditions du Rocher.

Piaget, J. (Ed.), 1967. Logique et connaissance scientifique, Paris, Gallimard.

Piaget, J., 1970. L'épistémologie génétique, Paris, Presses universitaires de France.

Wunenburger, J.-J., 1990. La raison contradictoire. Sciences et philosophie modernes : la pensée du complexe, Paris, Albin Michel. 